\title{
CHARACTERIZATIONS OF SIMPLY CONNECTED ROTATIONALLY SYMMETRIC MANIFOLDS
}

BY

\author{
HYEONG IN CHOI
}

\begin{abstract}
We prove that a simply connected, complete Riemannian manifold $M$ is rotationally symmetric at $p$ if and only if the exponential image of every linear subspace of $M_{p}$ is a smooth, closed, totally geodesic submanifold of $M$. This result is in essence Schur's theorem at one point $p$, as it becomes apparent in the proof.
\end{abstract}

A Riemannian manifold $M$ is called rotationally symmetric at a point $p$ of $M$ if the isotropy subgroup at $p$ of the isometry group of $M$ is $O(n)$. Recently the rotationally symmetric manifolds received much attention as model spaces for comparison in Riemannian geometry because of the simplicity of the geometric structure. This point was well recognized and systematically developed by Greene and $\mathrm{Wu}$ [2].

The rotational symmetry condition is a fairly strong one. Therefore there are many clear-cut geometric consequences. The purpose of this paper is to discuss some of these properties, and to show that they in turn characterize the rotational symmetry. Throughout this paper, $M$ is assumed to be a connected, simply connected, complete Riemannian manifold of dimension $n \geqslant 3$.

$M$ is topologically very simple: $M$ is diffeomorphic to $\mathbf{R}^{n}$ or $S^{n}$. One of the interesting geometric properties of $M$ is the existence of closed, totally geodesic submanifolds. In fact the exponential image of any linear subspace of $M_{p}$ is a smooth, closed, totally geodesic submanifold. In Theorem 3, which is our main result, we shall prove that the converse is also true.

Theorem 3 can be viewed heuristically as follows: A totally geodesic submanifold may be envisioned as a fixed point set of some isometry in a certain infinitesimal sense, even though there may be no such isometry. Thus the assumption that the exponential image of any linear hyperplane is totally geodesic suggests that all reflections with respect to those image hypersurfaces look like isometries. Since the group $O(n)$ is generated by reflections, it is quite natural to suspect the validity of Theorem 3.

Theorem 3 is a version of Schur's theorem at one point, as it becomes visibly clear in the proof. Schur's theorem (e.g. see [3, p. 202]) says that if the sectional curvature of a connected Riemannian manifold $N$ of dimension $\geqslant 3$ depends only on the points of $N$, then $N$ is a space of constant curvature. The idea of the proof of Schur's theorem is to show that $X k=0$ for any vector $X$, where $k$ is the curvature function,

Received by the editors November 9, 1981 and, in revised form, January 26, 1982.

1980 Mathematics Subject Classification. Primary 53C25.

(C)1983 American Mathematical Society 0002-9947/82/0000-0105/\$02.25 
by utilizing the second Bianchi identity. The key step in the proof of Theorem 3 is to prove that the radial curvature depends only on the distance from $p$ by carefully analyzing the variation of the radial curvature in the direction perpendicular to the radial direction.

I would like to thank Professor $\mathrm{H}$. Wu for constant encouragement and many helpful suggestions.

Let us fix some notations. As usual, the Riemannian metric of $M$ is denoted by $\langle$,$\rangle or d s^{2} . D_{X} Y$ denotes the covariant derivative of the Riemannian metric. The curvature tensor is defined to be $R(X, Y) Z=-D_{X} D_{Y} Z+D_{Y} D_{X} Z+D_{[X, Y]} Z$. The geodesic distance between two points $p$ and $q$ is denoted by $d(p, q)$. For $X \in M_{q}, \gamma_{X}$ denotes the geodesic such that $\gamma_{X}(0)=q$, and $\gamma_{X}^{\prime}(0)=X$. Let $p \in M$. We define $\delta_{p}$ to be the distance from $O \in M_{p}$ to the first conjugate locus in $M_{p}$. Define $\tilde{B}_{\delta_{p}}=\left\{X \in M_{p}|| X \mid<\delta_{p}\right\}$.

Let $p$ be a fixed point, and let $U$ be a normal coordinate neighborhood of $p$. Then at any point $q \in U$ we define radial planes to be 2-dimensional subspaces of $M_{q}$ which are tangent to the unique minimizing geodesic from $p$ to $q$. The radial curvature at $q$ is the restriction of sectional curvature to radial planes at $q$. In our problem we are interested in $\tilde{B}_{\delta_{p}}$ and its exponential image. But unfortunately $\tilde{B}_{\delta_{p}}$ may contain cut points of $p$. Thus we shall adopt the following more convenient approach: It is clear that $\tilde{B}_{\delta_{p}}$ becomes a Riemannian manifold when equipped with the metric $\exp _{p}^{*} d s^{2}$, since $\exp _{p}: \tilde{B}_{\delta_{p}} \rightarrow M$ is nonsingular. A two-dimensional plane $\Pi$ of $\left(M_{p}\right)_{Y}$ is called a radial plane if $\Pi$ is tangent to the ray from 0 to $Y$. The radial curvature of $\tilde{B}_{\delta_{p}}$ is the sectional curvature restricted to radial planes. The sectional curvature is, of course, computed with respect to the metric $\exp _{p}^{*} d s^{2}$.

Let $M$ be rotationally symmetric at $p$. Then the following facts on $B_{\delta_{p}}$ with metric $\exp _{p}^{*} d s^{2}$ are well known (see [2, pp. 24-30]).

(1) On $\tilde{B}_{\delta_{p}}, \exp _{p}^{*} d s^{2}=d r^{2}+f(r)^{2} d \theta^{2}$, for some function $f$, where $(r, \theta)$ are the usual polar coordinates of $\mathbf{R}^{n}$, and $d \theta^{2}$ is the metric of the standard sphere $S^{n-1}$.

(2) The intersection of any linear subspace of $M_{p}$ with $\tilde{B}_{\delta_{p}}$ is a totally geodesic submanifold of $\tilde{B}_{\delta_{p}}$.

(3) The radial curvature of $\tilde{B}_{\delta_{p}}$ depends only on the distance $r$ from $O \in M_{p}$. Let $k$ denote the radial curvature function.

(4) Every Jacobi field $J$ perpendicular to the geodesic ray $\gamma$ which vanishes at $O \in M_{p}$ is of the form $J(t)=f(t) E(t)$, where $E(t)$ is a parallel vector field along $\gamma . f$ satisfies the Jacobi equation $f^{\prime \prime}(t)+k(t) f(t)=0$, with $f(0)=0$, and $f\left(\delta_{p}\right)=0$ if $\delta_{p}<\infty$.

Proposition 1. Let $M$ be a simply connected manifold which is rotationally symmetric at $p$. Then $M$ is diffeomorphic to $S^{n}$ or $\mathbf{R}^{n}$, depending on whether $M$ is compact or noncompact.

Proof. See [1, Theorem 3.2].

COROllary 2. Let $M$ be rotationally symmetric at $p$. Then the exponential image of any linear subspace of $M_{p}$ is a closed, smooth, totally geodesic submanifold of $M$. 
Proof. Let $S$ be a linear subspace of $M_{p}$. Using the argument in the proof of Proposition 1, it is easy to show that $\exp _{p} S$ is a smooth, closed submanifold of $M$. Let $\Phi: M \rightarrow M$ be an isometry fixing $p$ such that the fixed point set $\Phi_{*}: M_{p} \rightarrow M_{p}$ is $S$, then the fixed point set of $\Phi$ is $\exp _{p} S$. Thus $\exp _{p} S$ is totally geodesic.

THEOREM 3. Let $M$ be a connected, simply connected, complete Riemannian manifold of dimension $n \geqslant 3$. Let $p$ be a fixed point of $M$. Then the following are equivalent:

(1) $M$ is rotationally symmetric at $p$.

(2) The exponential image of any linear subspace of $M_{p}$ is a closed, smooth, totally geodesic submanifold of $M$.

(3) For any point $q \in M$ such that $d(p, q)<\delta_{p}$, and for any minimal geodesic $\gamma$ from $p$ to $q$, the sectional curvature of any plane $\Pi$ of $M_{q}$ tangent to $\gamma$ at $q$ depends only on $d(p, q)$.

(4) The Riemannian metric pulled back under $\exp _{p}: M_{p} \rightarrow M$ is $\exp _{p}^{*} d s^{2}=d r^{2}+$ $f(r)^{2} d \theta^{2}$ on $\tilde{B}_{\delta_{p}}-\{0\}$ for some function $f$, where $(r, \theta)$ are the usual polar coordinates of $\mathbf{R}^{n}$, and $d \theta^{2}$ is the metric of the standard sphere.

REMARK. Considering $\tilde{B}_{\delta_{p}}$ as a manifold equipped with the Riemannian metric $\exp _{p}^{*} d s^{2}$, the condition (3) is equivalent to the following:

(3)' The radial curvature of $\tilde{B}_{\delta_{p}}$ in terms of the Riemannian metric $\exp _{p}^{*} d s^{2}$ depends only on $r$.

Proof. By Corollary 2, (1) implies (2). We will prove that (2) implies (3)'. Since our argument below is local, we may assume for simplicity that $\exp _{p}: \tilde{B}_{\delta_{p}} \rightarrow \exp \left(\tilde{B}_{\delta_{p}}\right)$ is a diffeomorphism. Define $\Omega=\exp _{p}\left(\tilde{B}_{\delta_{p}}\right)$. Let $q \in \Omega, q \neq p$, and let $\gamma$ be the minimizing geodesic from $p$ to $q$. Let $X, Y$, and $Z$ be O.N. vectors of $M_{q}$ such that $X$ is tangent to $\gamma$ at $q$. By assumption we can take the two-dimensional totally geodesic subspace $P$ tangent to $X$ and $Y$ which is perpendicular to $Z$. Since $P$ is totally geodesic, $R(X, Y) X$ is tangent to $P$, thus $\langle R(X, Y) X, Z\rangle=0$. Let $W_{t}=(\cos t) Y+$ $(\sin t) Z$ be a one-parameter family of vectors in $M_{q}$ which is perpendicular to $X$. Then $\dot{W}_{t}=-(\sin t) Y+(\cos t) Z$ is perpendicular to both $X$ and $W_{t}$. Thus $d\left\langle R\left(X, W_{t}\right) X, W_{t}\right\rangle / d t=2\left\langle R\left(X, \dot{W}_{t}\right) X, W_{t}\right\rangle=0$ by the same argument as above. This shows that the radial curvature depends only on the point $q \in M$. Let $k$ be the radial curvature function. We shall prove that $k$ depends only on the distance from $p$. Let $q, X, Y$ and $Z$ be defined as above. Since the exponential image of any linear subspace of $M_{p}$ is totally geodesic, by restricting to the image of a three-dimensional subspace of $M_{p}$, we may assume $\operatorname{dim} M=3$. Take the geodesic sphere $S$ centered at $p$ with radius $r=d(p, q)$. Extend $X$ to be the unit radial vector field near $q$. Take two totally geodesic two-dimensional surfaces $P_{1}$ and $P_{2}$ tangent to $Y$ and $Z$, respectively, and both tangent to $X$. Let $S_{1}$ and $S_{2}$ be the intersection of $S$ with $P_{1}$ and $P_{2}$, respectively. Extend $Y$ on $P_{2}$ as the unit normal vector field and along $S_{1}$ as the unit tangent vectors. Similarly extend $Z$ on $P_{1}$ and along $S_{2}$. The unit normal vector field on a totally geodesic hypersurface is globally parallel along any curve on the totally geodesic hypersurface. Thus we get $D_{X} Y \equiv 0$ on $P_{2}$ and $D_{X} Z \equiv 0$ on $P_{1}$. Also $D_{Y} Z \equiv 0$ along $S_{1}$, and $D_{Z} Y \equiv 0$ along $S_{2}$. We also have $D_{X} X \equiv 0$. Now $\left\langle D_{Y} X, X\right\rangle \equiv \frac{1}{2} Y\langle X, X\rangle \equiv 0$ along $S_{1}$, and $\left\langle D_{Y} X, Z\right\rangle \equiv Y\langle X, Z\rangle-\left\langle X, D_{Y} Z\right\rangle \equiv 0$ 
along $S_{1}$. Thus we have $D_{Y} X \equiv f Y$ along $S_{1}$ for some function $f$ defined on $S_{1}$. Also $\left\langle D_{Y} Y, Y\right\rangle \equiv \frac{1}{2} Y\langle Y, Y\rangle \equiv 0$ along $S_{1}$, and $\left\langle D_{Y} Y, Z\right\rangle \equiv Y\langle Y, Z\rangle-\left\langle Y, D_{Y} Z\right\rangle \equiv 0$ along $S_{1}$, and $\left\langle D_{Y} Y, X\right\rangle \equiv Y\langle Y, X\rangle-\left\langle Y, D_{Y} X\right\rangle \equiv-f$ along $S_{1}$. Thus we have $D_{Y} Y \equiv-f X$ along $S_{1}$.

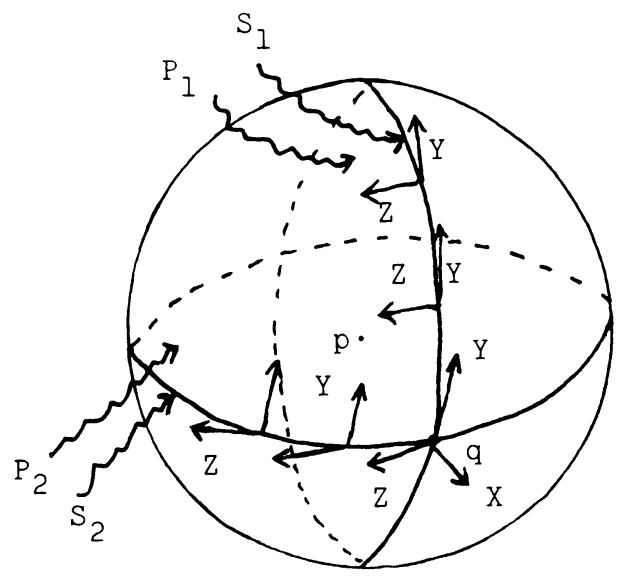

Similarly we have

$$
\begin{aligned}
& D_{Z} X \equiv g Z \quad \text { along } S_{2}, \\
& D_{Z} Z \equiv-g X \quad \text { along } S_{2} .
\end{aligned}
$$

Now $\langle R(X, Y) X, X\rangle \equiv 0$, and $\langle R(X, Y) X, Z\rangle \equiv\langle R(X, Z) X, Y\rangle \equiv 0$ along $S_{2}$, since $R(X, Z) X$ is tangent to $P_{2}$, and $Y$ is perpendicular to $P_{2}$. Thus $R(X, Y) X \equiv k Y$ along $S_{2}$. Similarly, $R(X, Z) X \equiv k Z$ along $S_{1}$. Take the family of geodesic spheres $S_{\alpha}$ of radius $\alpha$. Let $S_{1, \alpha}=S_{\alpha} \cap P_{1}$ and $S_{2, \alpha}=S_{\alpha} \cap P_{1}$. Note that $S_{1}=S_{1, r}$ and $S_{2}=S_{2, r}$. Extend $Y$ and $Z$ along $S_{1, \alpha}$ and $S_{2, \alpha}$, respectively, as unit tangent vector fields; then clearly $D_{Y} Z \equiv 0$ along $S_{1, \alpha}$ for any $\alpha$ close to $r$. Thus $D_{Y} Z \equiv 0$ along the geodesic ray from $p$ to $q$. Then since $R$ is a tensor, we get $R(X, Y) Z=-D_{X} D_{Y} Z+$ $D_{Y} D_{X} Z+D_{[X, Y]} Z=0$ at $q$, since $D_{Y} Z \equiv D_{X} Z \equiv 0$ and $[X, Y]$ is tangent to the totally geodesic $P_{1}$, and $Z$ is parallel on $P_{1}$. Similarly $R(Z, X) Y=0$ at $q$; then by the first Bianchi identity we get

$$
R(X, Y) Z=R(Z, X) Y=R(Y, Z) X=0 \text { at } q .
$$

For notational clarity, define $R(U, V, W)=R(U, V) W$ for vectors $U, V$ and $W$. We claim the following three equalities are true.

(i) $\left(D_{Z} R\right)(X, Y, X)=(Z k) Y$ at $q$.

(ii) $\left(D_{Y} R\right)(Z, X, X)=-(Y k) Z$ at $q$.

(iii) $\left(D_{X} R\right)(Y, Z, X)=0$ at $q$.

To prove (i), note that we already proved $R(X, Y) X \equiv k Y$ along $S_{2}$. Thus

$$
\begin{aligned}
\left(D_{Z} R\right)(X, Y, X)= & D_{Z}(R(X, Y) X)-R\left(D_{Z} X, Y\right) X \\
& -R\left(X, D_{Z} Y\right) X-R(X, Y) D_{Z} X .
\end{aligned}
$$

Now $D_{Z} Y=0$ implies the third term is zero, and $D_{Z} X=g Z$ and (*) imply the second and the fourth terms are zero. Thus we proved (i). Similarly we can prove (ii) 
just by interchanging $Y$ and $Z$. To prove (iii), it is easy to see that $R(Y, Z, X) \equiv$ $R(X, Z) X \equiv 0$ along the geodesic ray from $p$ to $q$ using the same argument to prove (*). Also $D_{X} Y=D_{X} Z=D_{X} X=0$ at $q$. Thus (iii) is proved. Adding (i), (ii) and (iii), and then applying the second Bianchi identity, we obtain $(Z k) Y-(Y k) Z=0$ at $q$. Since $Y$ and $Z$ are independent in $M_{q}$, we obtain $Z k=Y k=0$ at $q$. Since $q, Z$ and $Y$ are arbitrary, $k$ obviously depends only on the distance from $p$.

It is rather easy to prove that (3)' implies (4). Let $\gamma$ be a ray in $M_{p}$ from $O$. It can be easily seen that, with respect to the Riemannian metric $\exp _{p}^{*} d s^{2}$, the Jacobi field $J$ along $\gamma$, perpendicular to $\gamma$, is of the form $J(t)=f(t) E(t)$ for some function $f$, where $E$ is a vector field parallel along $\gamma$ and perpendicular to $\gamma . f$ satisfies the differential equation $f^{\prime \prime}(t)+k(t) f(t)=0$ with $f(0)=0$, and $f\left(\delta_{p}\right)=0$ if $\delta_{p}<\infty$. Thus (4) follows immediately.

Let us now assume (4). By applying the same Jacobi field argument, it is easy to see that the sphere in $M_{p}$ of radius $\delta_{p}$ is the first conjugate locus in $M_{p}$ of order $n-1$, if $\delta_{p}<\infty$. Again by Warner's theorem the first conjugate locus in $M_{p}$ and the cut locus in $M_{p}$ coincide, if $\delta_{p}<\infty$. Thus if $\delta_{p}<\infty$, the exponential map collapses the first conjugate locus in $M_{p}$ into a point $p^{*}$. Now the reflection with respect to any linear hyperplane in $M_{p}$ is an isometry with respect to the metric $\exp _{p}^{*} d s^{2}$, thus it defines an isometry of $M$ if $\delta_{p}=\infty$. If $\delta_{p}<\infty$, then it defines an isometry of $M-\left\{p^{*}\right\}$. But $p^{*}$ is a fixed point of the isometry, therefore it extends to $p^{*}$ as an isometry by continuity. Thus any reflection with respect to any linear hyperplane in $M_{p}$ is a differential of an isometry of $M$. Since the group $O(n)$ is generated by reflections, the isotropy subgroup at $p$ must be $O(n)$.

\section{REFERENCES}

1. C. Croke, Riemannian manifolds with large invariants, J. Differential Geom. 15 (1980), 467-491.

2. R. Greene and $\mathrm{H}$. Wu, Function theory on manifolds which possess a pole, Lecture Notes in Math., vol. 699, Springer-Verlag, Berlin and New York, 1979.

3. S. Kobayashi and K. Nomizu, Foundations of differential geometry, vol. I, Interscience, New York, 1963.

4. F. Warner, Conjugate loci of constant order, Ann. of Math. (2) 86 (1967), 192-212.

Department of Mathematics, University of California, Berkeley, California 94720

Current address: Department of Mathematics, University of Chicago, Chicago, Illinois 60637 